\title{
Dramatic Change in the Spectrum of FeLoBAL SDSS J163255.46+420407.7
}

\author{
Kentaro Aoki ${ }^{1}$ and Toru Misawa ${ }^{2}$ \\ ${ }^{1}$ Subaru Telescope, National Astronomical Observatory of Japan, \\ 650 North A'ohoku Place, Hilo, HI 96720, USA \\ Email: kaoki@subaru.naoj.org \\ ${ }^{2}$ Institute of Physical and Chemical Research (RIKEN), \\ 2-1, Hirosawa, Wako-si, Saitama, 351-0198, Japan
}

\begin{abstract}
We serendipitiously found that the iron-rich low-ionization broad absorption-line (FeLoBAL) quasar SDSS J 163255.46+420407.7 showed a dramatic change in its spectrum over 3.4 years in the rest frame of the quasar. A simple photoionization model suggests the increase in the nuclear radiation caused these changes. Given the recombination coefficient of $\mathrm{Fe}^{+}$, the relaxation time of the absorbing gas suggests $n_{e}>10^{4} \mathrm{~cm}^{-3}$.
\end{abstract}

Keywords. galaxies: active, quasars: individual (SDSS J 163255.46+420407.7), quasars: absorption lines

As shown in Figure 1, this quasar changed significantly between 2002 April and 2008 February when we observed it with Subaru/FOCAS. The spectrum taken in 2002 April by SDSS shows strong Fe II absorption lines while Fe II absorption became much weaker in our 2008 February spectrum. On the other hand, the equivalent widths of $\mathrm{Mg}$ II and He I* absorption have not changed very much. The continuum in 2008 became brighter than that in 2002 by factor of three at $2600 \AA$ in the quasar rest frame. The color of the continuum in 2008 also became bluer than that in 2002 , and matching quasar composite spectra.

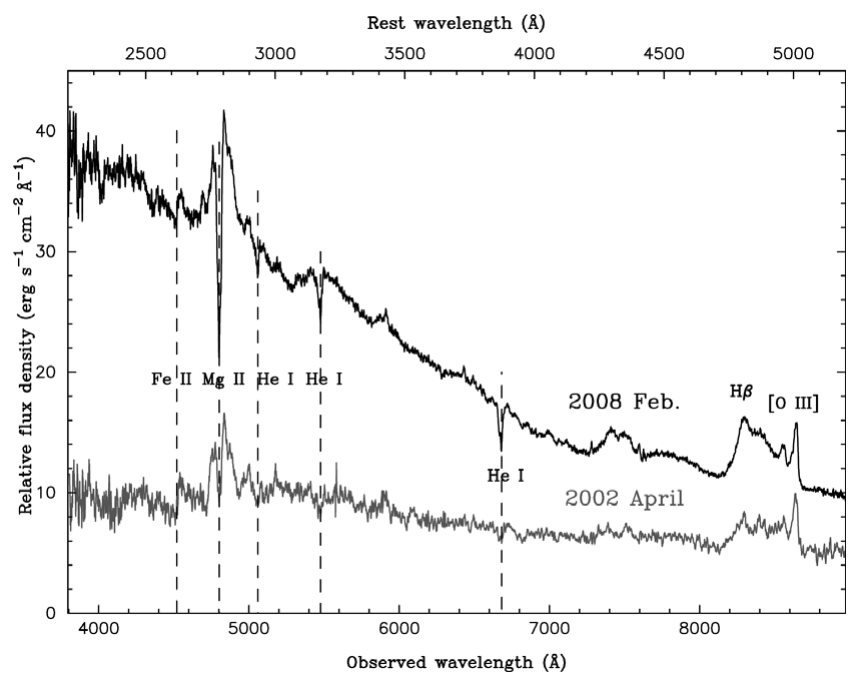

Figure 1. The spectrum taken with Subaru/FOCAS in 2008 February is shown in black, and that taken by SDSS in 2002 April is shown in grey. Both spectra are relatively flux calibrated assuming no change in [O III] emission-line flux. Vertical dashed lines indicate absoption lines. 